\title{
Periportal fibrosis without cirrhosis does not affect outcomes after continuous flow ventricular assist device implantation
}

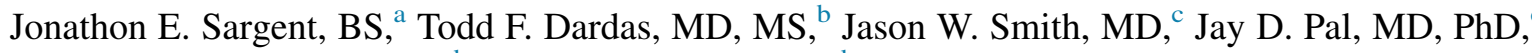 \\ Richard K. Cheng, MD, MS, ${ }^{b}$ S. Carolina Masri, MD, ${ }^{b}$ Kent R. Shively, BS, ${ }^{c}$ Lauren M. Colyer, BS, ${ }^{a}$ \\ Claudius Mahr, DO, ${ }^{\mathrm{b}}$ and Nahush A. Mokadam, MD
}

\begin{abstract}
Objective: This study investigates the relationship of periportal fibrosis on postoperative outcomes after ventricular assist device (VAD) implantation.

Methods: Between July 2005 and August 2014, a total of 233 patients were implanted with continuous flow VADs. Liver biopsy was performed on 16 patients with concern for liver disease. Survival was evaluated using the Kaplan-Meier method. The effect of fibrosis on length of stay (LOS) in the intensive care unit was modeled using Poisson regression. Adjustments were made for age, profile from the Interagency Registry for Mechanically Assisted Circulatory Support, biopsy, and model for end-stage liver disease score.

Results: Fourteen of the 16 patients who underwent biopsy had periportal fibrosis without cirrhosis. One-year survival for the groups with and without biopsy-proven fibrosis was $93 \% \pm 7 \%$ and $86 \% \pm 2 \%(P=.97)$, respectively. The intensive care unit LOS was not different for those with (median, 7 days; interquartile range: 3-14 days) versus without fibrosis (median, 6 days; interquartile range $4-10$ days; $P=.65)$. Fibrosis $(P=.42)$, age $(0.95)$, model for end-stage liver disease excluding internal normalized ratio-XI score $(P=.64)$, performance of a biopsy $(P=.28)$, and Interagency Registry for Mechanically Assisted Circulatory Support class $(P=.70)$ were not associated with intensive care unit LOS. Risk was increased of gastrointestinal bleeding $(14 \%$ vs $4 \% ; P=.026)$ in the first year among patients with fibrosis.
\end{abstract}

Conclusions: The presence of periportal fibrosis did not affect survival or outcomes in patients undergoing VAD implantation. These findings suggest that carefully selected patients with advanced heart failure and hepatic fibrosis without cirrhosis may achieve acceptable outcomes with VAD implantation. (J Thorac Cardiovasc Surg 2016;151:230-5)

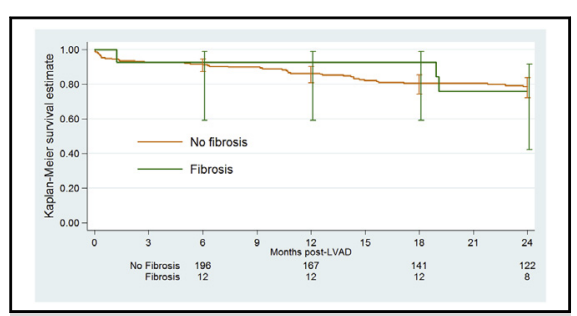

Kaplan-Meier survival curve of patients with documented fibrosis versus those without a biopsy.

\section{Central Message}

Selected patients with hepatic fibrosis without cirrhosis have acceptable outcomes with ventricular assist device implantation.

\section{Perspective}

Cirrhosis is often a contraindication for advanced heart failure therapies, but the periportal fibrosis on postoperative outcomes are not well characterized. Our results suggest that periportal fibrosis is not associated with worse outcomes after left ventricular assist device implantation. A reasonable approach is therefore to proceed with implantation in selected patients after careful evaluation.

See Editorial Commentary page 236.

See Editorials page 10 and 13.
From the ${ }^{\mathrm{a} S c h o o l}$ of Medicine, Divisions of ${ }^{\mathrm{b}}$ Cardiology, and ${ }^{\mathrm{c}}$ Cardiothoracic Surgery, University of Washington, Seattle, Wash.

Funding sources: American College of Cardiology/Daiichi Sankyo Career Development Award (to T.F.D.).

Read at the 95th Annual Meeting of The American Association for Thoracic Surgery, Seattle, Washington, April 25-29, 2015.

J.E.S. and T.F.D. contributed equally to this article.

Received for publication March 9, 2015; revisions received July 20, 2015; accepted for publication Aug 19, 2015; available ahead of print Sept 25, 2015.

Address for reprints: Nahush A. Mokadam, MD, Division of Cardiothoracic Surgery, University of Washington, 1959 NE Pacific, Box 356310, Seattle, WA 98195-6310 (E-mail: mokadamn@uw.edu).

$0022-5223 / \$ 36.00$

Copyright $(\odot 2016$ by The American Association for Thoracic Surgery

http://dx.doi.org/10.1016/j.jtcvs.2015.08.073
The current prevalence of heart failure in the United States is estimated to be at least 5.1 million people. ${ }^{1-3}$ Treatment for heart failure is dependent on the underlying etiology and may include pharmacologic therapies, conventional cardiac surgery, heart transplantation, or mechanical circulatory support (MCS), of which ventricular assist devices (VADs) are the most common. ${ }^{4,5}$ The risks of VAD implantation are multifactorial and include preoperative patient condition, coexisting comorbidities, hemodynamic state, intraoperative course, and postoperative management. ${ }^{6}$ Therefore, careful patient selection and optimization prior to surgery ensure the best outcomes. 


\section{Abbreviations and Acronyms \\ INR = international normalized ratio \\ INTERMACS $=$ Interagency Registry for Mechanically Assisted Circulatory Support \\ LOS = length of stay \\ MCS $\quad=$ mechanical circulatory support \\ MELD = model for end-stage liver disease \\ $\mathrm{VAD}=$ ventricular assist device}

End organ dysfunction, specifically hepatic dysfunction, can be a major complication of heart failure, and leads to increased mortality. ${ }^{7-10}$ Advanced heart failure therapies, such as cardiac transplantation and VAD implantation, are relatively contraindicated in patients with severe liver disease. Cardiac surgery has been empirically accepted to be contraindicated in patients with hepatic cirrhosis, but more data are needed to support this assumption. ${ }^{11}$ Additional needs are granular characterization of cirrhosis severity, and determination of the association of severity with perioperative outcomes. For example, severe hepatic cirrhosis (Child-Turcotte-Pugh, class C) has been shown to unacceptably increase mortality after cardiac surgery; whereas patients with class A cirrhosis have acceptable outcomes. ${ }^{7}$

Recently, the model for end-stage liver disease (MELD) scores has been shown to predict VAD perioperative transfusion requirements and operative mortality. ${ }^{12,13}$ However, the causes of hepatic dysfunction in patients with heart failure are likely multifactorial, and MELD scores fail to distinguish reversible from irreversible hepatopathy and were designed for use in patients with proven cirrhosis. In cases of reversible hepatopathy, VAD implantation may improve hepatic function to the point at which heart transplantation is possible. ${ }^{14,15}$ This study seeks to determine the effects of hepatic fibrosis without cirrhosis on outcomes after VAD implantation for the treatment of heart failure.

\section{METHODS}

\section{Design and Definition of Variables}

We retrospectively reviewed patients with advanced heart failure who received a primary VAD at our institution. Approval for this study was obtained from our institutional review board. Patients undergoing VAD exchange were excluded from this analysis. Patients with biopsy-proven hepatic fibrosis without cirrhosis were compared with the remainder of patients for demographics, preimplant characteristics, intensive care unit (LOS) and hospital LOS after VAD implantation, intraoperative course, postoperative characteristics, and overall survival. Indications for liver biopsy included abnormal imaging, transaminitis, chronically elevated right-sided filling pressures, and other factors deemed appropriate by the care team (Table 1).

The information and measurements collected for this patient population include demographic traits, laboratory data, preimplant hemodynamics, profile from the Interagency Registry of Mechanically Assisted Circulatory Support (INTERMACS), intraoperative details, postoperative outcomes, survival, and Model for End-Stage Liver Disease, excluding internationa normalized ratio (INR) (MELD-XI) score. The MELD-XI score excludes the INR, to eliminate the effects of anticoagulation, and thus may provide a better prognostic marker for patients on warfarin. ${ }^{16}$ For preoperative variables, data are reported as time closest to implant and within 30 days of implant.

\section{Study Population}

We reviewed 233 patients with heart failure who received VAD treatment at our institution between July 2005 and August 2014. Sixteen of these patients met clinical criteria for liver biopsy, with abnormal liver imaging being the most common indication. Biopsy samples were prepared by hematoxylin and eosin staining with additional trichrome staining. Fourteen patients were found to have hepatic fibrosis without cirrhosis, and 2 of the biopsied patients had normal liver architecture. Two of the patients, 1 who had fibrosis and 1 who did not, received isolated durable right VADs. Patients with documented cirrhosis were considered ineligible for advanced heart failure therapies, and they were not included in our database.

\section{Statistical Analysis}

All statistical analysis was conducted using STATA, version 13.1 (Stata Corporation, College Station, Tex). Comparisons of categoric variables were made using the Fisher exact test. Continuous variables were compared using the Student $t$ test or ANOVA. For all time-to-event analyses, patients were followed from implant until death without censoring for intervening VAD exchange, explant heart transplant, or the administrative censoring date of January 1, 2015. Survival analysis was conducted using the Kaplan-Meier method. The effects of hepatic fibrosis on intensive care unit LOS were modeled using Poisson regression, adjusting for age, INTERMACS profile, performance of a biopsy, and MELD-XI score.

\section{RESULTS}

\section{Demographics}

The demographics and baseline laboratory values are listed in Table 2. A total of 114 patients received heart transplants, and 46 died during the course of follow-up. The mean MELD-XI score for biopsied patients was $13.2 \pm 2.9$, and for those without biopsy, it was $14.4 \pm 4.4$. The higher MELD-XI scores were primarily attributable to higher creatinine values in the nonbiopsied patients ( $1.4 \pm 0.6$ vs $1.2 \pm 0.4)$. The mean MELD-XI score for the group without documented fibrosis was $14.4 \pm 4.4$; for the group with fibrosis, it was $13.7 \pm 2.7$.

The group without documented fibrosis was $18.3 \%$ women; the group with fibrosis, $28.6 \%$ women. The mean age for the group without documented fibrosis was $52.8 \pm 14.6$ years; for the group with fibrosis, $55.1 \pm 8.8$ years. The group without documented fibrosis had a mean body mass index of $27.7 \pm 6.2 \mathrm{~kg} / \mathrm{m}^{2}$; the group with fibrosis, $27.1 \pm 6.0 \mathrm{~kg} / \mathrm{m}^{2}$.

\section{Preoperative Characteristics}

Preoperative characteristics are listed in Table 2. No statistically significant difference was found in preoperative 
TABLE 1. Indications for liver biopsy

\begin{tabular}{lc}
\hline \multicolumn{1}{c}{ Indication } & Pre-VAD biopsy $(\mathbf{n}=\mathbf{1 6})$ \\
\hline Elevated total bilirubin & 10 \\
CVP $>15$ & 9 \\
Abnormal ultrasound & 9 \\
Ascites & 6 \\
Elevated AST/ALT & 6 \\
Abnormal CT & 5 \\
Hepatitis B/C + & 5 \\
Alcohol abuse & 4 \\
Alpha-1-antitrypsin deficiency & 2 \\
Several patients had >1 indication for biopsy. $V A D$, Ventricular assist device; \\
$C V P$, central venous pressure; AST, aspartate aminotransferase; $A L T$, alanine \\
aminotransferase; $C T$, computed tomography.
\end{tabular}

laboratory values between the group with fibrosis and the group without documented fibrosis. Of the measurements of hepatic dysfunction, mean alkaline phosphatase was elevated in the group with fibrosis, compared with the group without documented fibrosis (126.2 vs 86.8 units/L, $P=.001)$, as was mean platelet count $\left(231 \mathrm{vs} 191 \times 10^{3} /\right.$ $\mu \mathrm{L}, P=.042)$. The only statistically significant difference in right heart catheterization data between the 2 groups was a higher mean cardiac index, as measured by thermodilution found in the group with fibrosis, compared with the group without documented fibrosis (2.7 vs $\left.2.3 \mathrm{~L} / \mathrm{min} / \mathrm{m}^{2}, P=.025\right)$.

\section{Intraoperative Details}

Intraoperative details are described in Table 3. No difference was found between the 2 groups for the following: cardiopulmonary bypass and crossclamp times; percentage of patients undergoing reoperative cardiac surgery; concomitant procedures and use of right VAD therapy after left VAD implantation $(P=1.00)$.

\section{Postoperative Outcomes}

Postoperative outcomes are listed in Table 4. The overall hospital LOS was not statistically different for patients with fibrosis, compared with those without documented fibrosis, either before (23.5 vs 19.0 days; Poisson regression fibrosis coefficient $0.15, P=.36$ ) or after adjustment (fibrosis coefficient $0.15 ; P=.35$ ) for age, MELD-XI score, performance of biopsy, and INTERMACS class. No statistical difference was present in ICU LOS between the 2 groups before (fibrosis median days $=7.0$, nondocumented fibrosis median days $=6.0$; Poisson regression coefficient $0.12, P=.65$ ) versus after adjustment (beta coefficient for fibrosis is $0.12 ; P=.64$ ) for age, MELD-XI score, performance of a biopsy, and INTERMACS class. Similarly, the 2 groups did not differ in incidence of reintubation $(P=.652)$, renal failure $(P=.265)$, dialysis $(P=.325)$, or VAD infection within 1 year $(P=.765)$ after implantation. A statistically significant difference was found in the likelihood of gastrointestinal bleeding in the year after VAD (14\% vs $4 \% ; P=.026)$, with higher bleeding among patients with fibrosis.

\section{Survival}

Thirty-day survival for the group without documented fibrosis was $95 \%$; for the group with fibrosis, it was $100 \%$. One-year survival for the groups with and without documented fibrosis was $93 \% \pm 7 \%$ and $86 \% \pm 3 \%$, respectively. The presence of periportal fibrosis was not associated with higher mortality $(P=.97$; Figure 1 , Table 4). A history of liver biopsy was not associated with differential survival $(P=.82)$.

\section{DISCUSSION}

The use of VAD therapy as a treatment for heart failure refractory to medical therapy is increasing. As the prevalence of VAD implantation rises, proper patient selection becomes increasingly important, and predictors of outcome and contraindications for the procedure need to be more accurately described. Our study sought to accomplish this description by determining the effects of periportal fibrosis without cirrhosis on postoperative outcome after VAD implantation.

The MELD scores, originally intended to assess mortality in cirrhotic patients undergoing transjugular intrahepatic portosystemic shunt procedures, ${ }^{17,18}$ have been shown to be useful prognostic indicators in cardiac and noncardiac procedures. Specifically, MELD scores were recently shown to predict transfusion requirements and in-hospital mortality after VAD implantation ${ }^{12,13}$ and transplant. ${ }^{10}$ In the context of multiple organ system failure, such as heart failure, MELD scores are likely to be less representative of intrinsic hepatic dysfunction and more likely to reflect the extent of heart failure. For example, impaired cardiac output, cardiorenal syndrome, and congestive hepatopathy will contribute to elevated serum creatinine, 1 of 3 variables used to calculate MELD scores.

Contrary to previous reports, ${ }^{16,18}$ our study found no association between MELD-XI score and survival after VAD implantation for treatment of heart failure. A possible explanation for this discrepancy is our use of MELD-XI scores, which eliminates INR from the equation to account for anticoagulation. Given evidence that VAD implantation has been shown to improve reversible liver disease in patients with heart failure, ${ }^{14}$ and conflicting reports of association between MELD and survival in patients treated with VAD implantation for heart failure, the utility of MELD scores for predicting outcomes remains unclear.

Liver biopsy is commonly performed to exclude cirrhosis, which is considered a contraindication for 
TABLE 2. Demographics and baseline characteristics

\begin{tabular}{|c|c|c|c|}
\hline Demographics & $\begin{array}{l}\text { Documented } \\
\text { fibrosis absent } \\
\quad(\mathbf{n}=\mathbf{2 1 9})\end{array}$ & $\begin{array}{l}\text { Documented } \\
\text { fibrosis present } \\
(n=14)\end{array}$ & $\begin{array}{c}P \\
\text { value }\end{array}$ \\
\hline Gender, female $(\%)$ & 18.30 & 28.60 & .308 \\
\hline Age (y) & $52.8 \pm 14.6$ & $55.1 \pm 8.8$ & .549 \\
\hline Body mass index $\left(\mathrm{kg} / \mathrm{m}^{2}\right)$ & $27.7 \pm 6.1$ & $27.1 \pm 6.0$ & .740 \\
\hline $\begin{array}{l}\text { Blood pressure (systolic) } \\
\text { (mm Hg) }\end{array}$ & $105.4 \pm 13.9$ & $108.2 \pm 15.4$ & .498 \\
\hline $\begin{array}{l}\text { Blood pressure (diastolic) } \\
\text { (mm Hg) }\end{array}$ & $68.9 \pm 10.9$ & $69.5 \pm 7.4$ & .852 \\
\hline Pre-LVAD liver biopsy (n) & 2 & 14 & \\
\hline MELD-XI (units) & $14.3 \pm 4.4$ & $13.7 \pm 2.7$ & .582 \\
\hline INTERMACS Profile & & & .698 \\
\hline 1 & 19 & 0 & \\
\hline 2 & 64 & 5 & \\
\hline 3 & 90 & 7 & \\
\hline 4 & 21 & 0 & \\
\hline 5 & 13 & 1 & \\
\hline 6 & 9 & 1 & \\
\hline 7 & 3 & 0 & \\
\hline Year of implant & & & .423 \\
\hline 2005 & 5 & 0 & \\
\hline 2006 & 4 & 0 & \\
\hline 2007 & 7 & 0 & \\
\hline 2008 & 15 & 4 & \\
\hline 2009 & 23 & 0 & \\
\hline 2010 & 30 & 1 & \\
\hline 2011 & 39 & 2 & \\
\hline 2012 & 34 & 3 & \\
\hline 2013 & 34 & 3 & \\
\hline 2014 & 28 & 1 & \\
\hline \multicolumn{4}{|l|}{ Laboratory tests } \\
\hline Serum sodium $(\mathrm{mEq} / \mathrm{L})$ & $132.7 \pm 4.3$ & $132.6 \pm 4.3$ & .912 \\
\hline Blood urea nitrogen $(\mathrm{mg} / \mathrm{dL})$ & $25.0 \pm 16.9$ & $31.6 \pm 24.3$ & .167 \\
\hline Serum creatinine $(\mathrm{mg} / \mathrm{dL})$ & $1.4 \pm 0.6$ & $1.3 \pm 0.4$ & .279 \\
\hline Hematocrit $(\%)$ & $32.6 \pm 5.1$ & $32.6 \pm 4.3$ & .963 \\
\hline Platelets $(\times 1000 / \mu 1)$ & $191.1 \pm 70.0$ & $231.4 \pm 90.8$ & .042 \\
\hline Albumin $(\mathrm{gm} / \mathrm{dL})$ & $3.2 \pm 0.5$ & $3.3 \pm 0.4$ & .450 \\
\hline AST (units/L) & $37.8 \pm 29.9$ & $29.3 \pm 7.8$ & .288 \\
\hline ALT (units/L) & $45.7 \pm 70.0$ & $25.1 \pm 12.2$ & .275 \\
\hline Total bilirubin (mg/dL) & $1.4 \pm 0.8$ & $1.5 \pm 0.4$ & .740 \\
\hline $\begin{array}{l}\text { Alkaline phosphatase } \\
\text { (units/L) }\end{array}$ & $86.8 \pm 40.4$ & $126.2 \pm 60.1$ & .001 \\
\hline \multicolumn{4}{|l|}{ Hemodynamic values } \\
\hline $\begin{array}{l}\text { Right atrial pressure } \\
\quad(\mathrm{mm} \mathrm{Hg})\end{array}$ & $10.1 \pm 5.7$ & $10.2 \pm 5.7$ & .945 \\
\hline $\begin{array}{l}\text { Right ventricle systolic } \\
\text { pressure }(\mathrm{mm} \mathrm{Hg})\end{array}$ & $47.2 \pm 14.2$ & $39.5 \pm 10.2$ & .054 \\
\hline $\begin{array}{l}\text { Right ventricle diastolic } \\
\text { pressure }(\mathrm{mm} \mathrm{Hg})\end{array}$ & $10.8 \pm 6.0$ & $9.5 \pm 5.3$ & .454 \\
\hline $\begin{array}{l}\text { Mean pulmonary artery } \\
\text { pressure }(\mathrm{mm} \mathrm{Hg})\end{array}$ & $32.6 \pm 9.3$ & $27.7 \pm 8.5$ & .069 \\
\hline $\begin{array}{l}\text { Pulmonary capillary wedge } \\
\text { pressure }(\mathrm{mm} \mathrm{Hg})\end{array}$ & $22.5 \pm 7.9$ & $19.8 \pm 6.2$ & .234 \\
\hline $\begin{array}{l}\text { Cardiac output } \\
\text { (thermodilution) (L/min) }\end{array}$ & $4.7 \pm 1.4$ & $5.3 \pm 1.3$ & .146 \\
\hline
\end{tabular}

TABLE 2. Continued

\begin{tabular}{|c|c|c|c|}
\hline Demographics & $\begin{array}{c}\text { Documented } \\
\text { fibrosis absent } \\
(\mathbf{n}=\mathbf{2 1 9}) \\
\end{array}$ & $\begin{array}{l}\text { Documented } \\
\text { fibrosis present } \\
\quad(n=14) \\
\end{array}$ & $\begin{array}{r}P \\
\text { value } \\
\end{array}$ \\
\hline $\begin{array}{l}\text { Cardiac index } \\
\text { (thermodilution) } \\
\left(\mathrm{L} / \mathrm{min} / \mathrm{m}^{2}\right)\end{array}$ & $2.3 \pm 0.6$ & $2.7 \pm 0.6$ & .025 \\
\hline $\begin{array}{l}\text { Cardiac output (Fick) } \\
\text { (L/min) }\end{array}$ & $4.4 \pm 1.3$ & $4.6 \pm 1.7$ & .628 \\
\hline $\begin{array}{l}\text { Cardiac index (Fick) } \\
\left(\mathrm{L} / \mathrm{min} / \mathrm{m}^{2}\right)\end{array}$ & $2.1 \pm 0.6$ & $2.4 \pm 0.7$ & .251 \\
\hline Heart rate (beats/min) & $88.4 \pm 16.6$ & $90.7 \pm 16.3$ & .621 \\
\hline
\end{tabular}

advanced heart failure therapies. The results of these biopsies, even in patients without cirrhosis, may influence the decision to offer or withhold VAD therapy. Further investigation is needed to fully describe the role that liver disease of varying severities plays on mortality after advanced heart failure therapies. Concern remains that periportal fibrosis without cirrhosis would adversely affect outcomes after VAD implantation. Our results suggest that periportal fibrosis without cirrhosis is not strongly associated with perioperative or most short-term postoperative outcomes.

An association was found between fibrosis and increased gastrointestinal bleeding after VAD implantation. This association was not correlated with increased mortality and warrants further investigation. Additionally, presence of periportal fibrosis does not increase average length of stay in the hospital or intensive care unit. Periportal fibrosis may be more reflective of congestive (and thus reversible) hepatopathy than of structural liver disease. ${ }^{19}$ Therefore, patients with heart failure and periportal fibrosis without cirrhosis may benefit from VAD implantation for treatment of advanced heart failure, and VAD

TABLE 3. Intraoperative details

\begin{tabular}{lccr}
\hline \multicolumn{1}{c}{ Variable } & $\begin{array}{c}\text { Documented } \\
\text { fibrosis absent } \\
(\mathbf{n}=\mathbf{2 1 9})\end{array}$ & $\begin{array}{c}\text { Documented } \\
\text { fibrosis present } \\
(\mathbf{n}=\mathbf{1 4})\end{array}$ & $\begin{array}{c}\boldsymbol{P} \\
\text { value }\end{array}$ \\
\hline Reoperation (n [\%]) & $52(23.74)$ & $2(14.3)$ & .531 \\
CPB time (min) & $94.7 \pm 44.5$ & $83.1 \pm 34.9$ & .344 \\
CC time (min) & $4.6 \pm 16.0$ & $5.9 \pm 15.1$ & .762 \\
Additional procedures & & & \\
$\quad$ TV repair & $41(18.7)$ & $2(14.3)$ & 1.000 \\
PFO closure & $34(15.5)$ & $1(7.1)$ & .700 \\
Aortic valve procedure & $5(2.3)$ & $0(0)$ & 1.000 \\
RVAD & $5(2.3)$ & $0(0)$ & 1.000 \\
\hline
\end{tabular}

Values are $\mathrm{n}(\%)$, or mean $\pm \mathrm{SD}$, unless otherwise indicated. $C P B$, Cardiopulmonary bypass; $C C$, crossclamp; $T V$, tricuspid valve; $P F O$, patent foramen ovale; $R V A D$, right ventricular assist device. 
TABLE 4. Postoperative results

\begin{tabular}{lccc}
\hline \multicolumn{1}{c}{ Outcome } & $\begin{array}{c}\text { Documented } \\
\text { fibrosis absent } \\
(\mathbf{n}=\mathbf{2 1 9})\end{array}$ & $\begin{array}{c}\text { Documented } \\
\text { fibrosis present } \\
(\mathbf{n}=\mathbf{1 4})\end{array}$ & $\begin{array}{c}\boldsymbol{P} \\
\text { value }\end{array}$ \\
\hline 1-y survival (\%) & $86 \pm 2$ & $93 \pm 7$ & .97 \\
Postoperative hospital LOS (d) & $23.4 \pm 15.3$ & $27.1 \pm 16.3$ & .375 \\
ICU LOS (d) & $8.8 \pm 9.9$ & $9.9 \pm 9.4$ & .691 \\
Reintubated & $22(10.5)$ & $2(14.3)$ & .652 \\
Renal failure & $14(6.7)$ & $2(14.3)$ & .265 \\
Dialysis (new) & $5(2.4)$ & $1(7.1)$ & .325 \\
VAD infection within 1 y & $59(26.9)$ & $3(21.4)$ & .765 \\
$\quad$ Driveline infection & $39(18.2)$ & $3(21.4)$ & .726 \\
$\quad$ Pump pocket infection & $23(10.5)$ & $0(0)$ & .372 \\
Gastrointestinal bleeding & $37(16.9)$ & $6(42.9)$ & .026 \\
$\quad$ within 1 y & & & \\
\hline
\end{tabular}

Values are $\mathrm{n}(\%)$, or mean $\pm \mathrm{SD}$, unless otherwise indicated. LOS, Length of stay; $I C U$, intensive care unit; $V A D$, ventricular assist device.

should not be withheld on the basis of periportal fibrosis alone.

Our study was limited to patients who underwent VAD implantation. Certain patients may not have been given the option of this surgery because they were at higher risk owing to liver-related issues, or to a combination of factors that may have included fibrosis. Other limitations of this study are the small sample size and the limited statistical power. A greater number of patients who undergo liver biopsies are needed to validate these findings. In addition, further investigation is needed to better characterize the effects of liver disease on mortality after VAD implantation.

This study was retrospective and is confined by the typical limitations of such studies. Further, the study was nonrandomized, and not all patients underwent biopsy. Patients who did not undergo biopsy may exhibit a degree of hepatic dysfunction that was not clinically suspected. Our current criteria for proceeding with liver biopsy include abnormal imaging, abnormal liver function tests, chronically elevated right-sided filling pressures, and any stigmata of chronic liver disease.

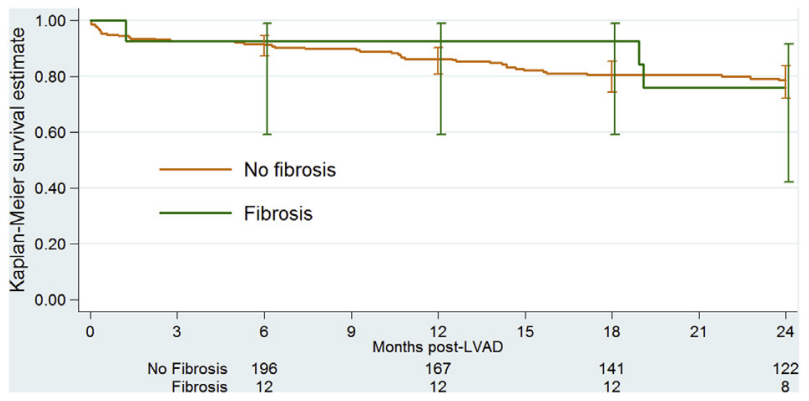

FIGURE 1. Kaplan-Meier survival curve of patients with documented fibrosis versus those without a biopsy. $L V A D$, Left ventricular assist device.
In a multivariable model including MELD-XI score, INTERMACS profile, performance of a biopsy, presence of periportal fibrosis, and age, only progressive age is associated with increased mortality after VAD implantation for treatment of heart failure. For many patients with heart failure, liver disease remains a large barrier to advanced heart failure therapies. However, the spectrum of liver disease and its effects on postoperative outcomes after advanced heart failure therapies are not well characterized. Neither presence of periportal fibrosis nor performance of a biopsy was associated with increased mortality after VAD implantation for treatment of heart failure. This finding was unexpected and reassuring, given that liver disease is frequently considered a contraindication to cardiac surgery.

Based on this observation, we have changed our practice to more liberally consider patients who have periportal fibrosis, but do not have cirrhosis, for advanced heart failure therapies. Carefully selected patients with liver dysfunction may benefit from VAD implantation.

\section{Conflict of Interest Statement}

Dr Pal reports a relationship with Smith and Nephew. Dr Mahr is a consultant to Thoratec Corporation, HeartWare, and Abiomed. Dr Mokadam is a consultant to Thoratec Corporation, HeartWare, and St Jude Medical, Inc. The other authors have nothing to disclose with regard to commercial support.

\section{References}

1. Cowie MR, Mosterd A, Wood DA, Deckers JW, Poole-Wilson PA, Sutton GC, et al. The epidemiology of heart failure. Eur Heart J. 1997;18:208.

2. Hoes AW, Mosterd A, Grobbee DE. An epidemic of heart failure? Recent evidence from Europe. Eur Heart J. 1998;19(Suppl L):L2.

3. Go AS, Mozaffarian D, Roger VL, Benjamin EJ, Berry JD, Borden WB, et al. Heart disease and stroke statistics - 2013 update: a report from the American Heart Association. Circulation. 2013;127:e6.

4. McMurray JJ, Adamopoulos S, Anker SD, Auricchio A, Böhm M, Dickstein K, et al. ESC Guidelines for the diagnosis and treatment of acute and chronic heart failure 2012: The Task Force for the Diagnosis and Treatment of Acute and Chronic Heart Failure 2012 of the European Society of Cardiology. Developed in collaboration with the Heart Failure Association (HFA) of the ESC. Eur Heart J. 2012;33:1787.

5. Yancy CW, Jessup M, Bozkurt B, Butler J, Casey DE, Drazner MH, et al. 2013 ACCF/AHA guideline for the management of heart failure: executive summary: a report of the American College of Cardiology Foundation/American Heart Association Task Force on practice guidelines. Circulation. 2013;128: 1810-52.

6. Takeda K, Takayama H, Kalesan B, Uriel N, Colombo PC, Jorde UP, et al. Long-term outcome of patients on continuous-flow left ventricular assist device support. J Thorac Cardiovasc Surg. 2014;148:1606-14.

7. Vanhuyse F, Maureira P, Portocarrero E, Laurent N, Lekehal M, Carteaux JP, et al. Cardiac surgery in cirrhotic patients: results and evaluations of risk factors. Eur J Cardiothorac Surg. 2012;42:293-9.

8. Yang JA, Kato TS, Shulman BP, Takayama H, Farr M, Jorde UP, et al. Liver dysfunction as a predictor of outcomes in patients with advanced heart failure requiring ventricular assist device support: use of the model of end-stage liver disease (MELD) and MELD eXcluding INR (MELD-XI) scoring system. J Heart Lung Transplant. 2012;31:601-10.

9. Kim MS, Kato TS, Farr M, Wu C, Givens RC, Collado E, et al. Hepatic dysfunction in ambulatory patients with heart failure: application of the 
MELD scoring system for outcome prediction. J Am Coll Cardiol. 2013;61: 2253-61.

10. Chokshi A, Cheema FH, Schaefle KJ, Jiang J, Collado E, Shahzad K, et al. Hepatic dysfunction and survival after orthotopic heart transplantation: application of the MELD scoring system for outcome prediction. J Heart Lung Transplant. 2012:31:591-600.

11. Bizouarn P, Ausseur A, Desseigne P, Le Teurnier Y, Nougarede B, Train M, et al. Early and late outcome after elective cardiac surgery in patients with cirrhosis. Ann Thorac Surg. 1999;67:1334-8.

12. Matthews JC, Pagani FD, Haft JW, Koelling TM, Naftel DC, Aaronson KD. Model for end-stage liver disease score predicts left ventricular device operative transfusion requirements, morbidity, and mortality. Circulation. 2010;121: 214-20.

13. Deo SV, Daly RC, Altarabsheh SE, Hasin T, Zhao Y, Shah IK, et al. Predictive value of the model for end-stage liver disease score in patients undergoing left ventricular assist device implantation. ASAIO J. 2013;59:57-62.

14. Demirozu ZT, Hernandez R, Mallidi HR, Singh SK, Radovancevic R, Segura AM, et al. HeartMate II left ventricular assist device implantation in patients with advanced hepatic dysfunction. J Card Surg. 2014;29:419-23.

15. Weymann A, Patil NP, Sabashnikov A, Mohite PN, Cargia Saez D, Bireta C, et al. Continuous-flow left ventricular assist device therapy in patients with preoperative hepatic failure: Are we pushing the limits too far? Artif Organs. 2015;39: 236-42.

16. Inohara T, Kohsaka S, Shiraishi Y, Goda A, Sawano M, Yagawa M, et al. Prognostic impact of renal and hepatic dysfunction on the MELD-XI score in patients with acute heart failure. Int J Cardiol. 2014;176:571-3.

17. Malinchoc M, Kamath PS, Gordon FD, Peine CJ, Rank J, Borg PC. A model to predict poor survival in patients undergoing transjugular intrahepatic portosystemic shunts. Hepatology. 2003;31:864-71.

18. Farr M, Mitchell J, Lippel M, Kato TS, Jin Z, Ippolito P, et al. Combination of liver biopsy with MELD-XI scores for post-transplant outcome prediction in patients with advanced heart failure and suspected liver dysfunction. J Heart Lung Transplant. 2015;34:873-82.

19. Ellis EL, Mann DA. Clinical evidence for the regression of liver fibrosis. J Hepatol. 2012;56:1171-80.

Key Words: heart failure, heart-assist devices, liver diseases, survival analysis

\section{Discussion}

Dr Y. Naka (New York, NY). Severe liver dysfunction that warrants the clinical decision to do a liver biopsy is a very important issue, but very little guidance is available in the literature. So this is a great study, and I would like to congratulate the authors. I have a few comments and questions.

The first one is: Periportal fibrosis is specific to zone-1 fibrosis in the liver pathology field, and donor fibrosis is predominant in bilateral hepatitis and amiodarone toxicity. The subject of concern in this study, such as advanced heart failure and then congestive hepatopathy, is usually predominantly associated with zone-3 fibrosis, which is called a centrilobular fibrosis. So have you looked at those issues? That is the first question.

The second question is: The etiology of liver disease is critically important. The sign of severe right-sided dysfunction, such as ascites, or persistent and severe edema or pleural effusion, or the history, or the current bilateral hepatitis, and amiodarone toxicity-all that information is important. Have you looked at those issues?

Dr Sargent. To address the first question, for the issue of temporizing the fibrosis to zone 1 or zone 3 , our pathologists and hepatologists used a more binary formula for describing fibrosis. Therefore, we do not have the data to further describe the fibrosis.

To address the second question, of the etiology of fibrosis in our patients: We do not have information about the specific etiology leading to the state of fibrosis. For the patients with fibrosis, however, we do have some data regarding their risk factors for fibrosis.

Dr Naka. The reason I ask that question is that the control group patients may have similar characteristics in terms of etiology, so that that needs to be further scrutinized. The third question is very quick: Can you advance a basic algorithm to determine which VAD patient should be considered for liver biopsy?

Dr Sargent. We have started to examine that information, and the more-specific indications for liver biopsy, so that is an ongoing analysis. 